J. Klin. Endokrinol. Stoffw. 2019 • 12:70-72

https://doi.org/10.1007/s41969-019-0068-5

ㄷ Der/die Autor(en) 2019

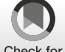

Eine Hypothyreose bedeutet die Unterversorgung des Organismus mit Schilddrüsenhormonen. Gerade in der pränatalen und frühen postnatalen Entwicklung ist die ausreichende Versorgung mit Schilddrüsenhormonen von besonderer Bedeutung. Die kongenitale Hypothyreose ist die häufigste vermeidbare Ursache mentaler Retardierung, ist aber auch mit einem ca. 12-fach erhöhten Risiko für multiple neonatale Missbildungen assoziiert, die weitere Komplikationen für die Erkrankten bedeuten. Daher ist ein entsprechendes Neugeborenen-Screeningprogramm eine äußerst wichtige Maßnahme zur Vermeidung zumindest der mentalen Retardierung aufgrund eines Schilddrüsenhormonmangels [1-4].

\section{Pathophysiologie und klinische Bedeutung}

Die kongenitale Hypothyreose, definiert als ein von Geburt an bestehender Schilddrüsenhormonmangel, kann weiter näher klassifiziert werden, indem zwischen einer primären (Schilddrüse ist direkt betroffen) oder einer sekundären, zentralen (Hypophyse oder Hypothalamus betroffen) kongenitalen Hypothyreose unterschieden wird. Zusätzlich kann auch hinsichtlich der Dauer (transient gegenüber permanent) differenziert werden. Da die zentrale sekundäre kongenitale Hypothyreose, die häufig mit einem TSH-Mangel und einer angeborenen Hypophysenvorderlappeninsuffizienz assoziiert ist, sehr selten vorkommt, wird im Rahmen dieses Beitrages lediglich die primäre kongenitale Hypothyreose näher beleuchtet.

Die transiente primäre kongenitale $\mathrm{Hy}$ pothyreose kann als Folge schweren Jodmangels insbesondere in Gebieten mit endemischem Jodmangel auftreten, kann

\title{
Sabina Baumgartner-Parzer
}

Universitätsklinik für Innere Medizin III, Medizinische Universität Wien, Wien, Österreich

\section{Primäre kongenitale Hypothyreose}

aber auch durch mütterliche Schilddrüsen-Autoantikörper, mütterliche Thyreostatikatherapie oder durch akuten Jodexzess hervorgerufen werden [4]. In der Mehrheit der Fälle (>80\%) liegt der permanenten primären kongenitalen Hypothyreose eine Entwicklungsstörung der Schilddrüse (Dysgenesie) zugrunde, während angeborene Störungen der Schilddrüsenhormon-Biosynthese (Dyshormonogenese) für ca. $10-15 \%$ der Fälle verantwortlich zeichnen (• Tab. 1).

Eine Dysgenesie ist charakterisiert durch eine Ektopie (Schilddrüsengewebe zwischen Zungengrund und vor der Luftröhre liegend), eine Athyreose oder eine Hypoplasie/Hemiagenesie der Schilddrüse aufgrund einer gestörten Embryonalentwicklung. In Gebieten mit ausreichender Jodversorgung stellt die Ektopie die häufigste Ursache $(70 \%$ der Fälle) dar, während eine Athyreose (komplettes Fehlen der Schilddrüse) in ca. $25 \%$ und die Hypoplasie/Hemiagenesie in ca. $5 \%$ der Fälle zu beobachten sind [1-6].

\section{Symptomatik und Diagnostik}

Lediglich bei einem geringen Prozentsatz der PatientInnen sind schon bei der Geburt charakteristische klinische Zeichen einer Hypothyreose zu beobachten. $\mathrm{Zu}$ Letzteren gehören Icterus prolongatus, große Fontanelle (vor allem posterior), Hypotonie, Adynamie, Trinkschwäche, kurze Wachphasen, rasche Gewichtszunahme, Hypothermie, Makroglossie, zunehmend grobe Gesichtszüge oder auch Umbilikalhernien. Verlangsamtes Größenwachstum und eine Entwicklungsverzögerung fallen meist erst später auf. Die klinischen Zeichen sind, möglicherweise auch durch den transplazentaren
Transport maternaler Schilddrüsenhormone bedingt, oft nur sehr gering ausgeprägt. Daher war die Einführung neonataler Screeningprogramme eine wichtige Maßnahme, da damit eine präklinische biochemische Diagnose, gefolgt von einer entsprechenden Bestätigungsdiagnostik, mit nachfolgender Substitutionstherapie in der ersten Lebenswoche möglich ist und somit eine zerebrale Schädigung und mentale Retardierung bei den betroffenen Kindern hintangehalten werden können [1-5].

\section{Genetische Diagnostik}

\section{Vererbung und Häufigkeit}

Die Prävalenz der kongenitalen Hypothyreose liegt bei ca. $2-4$ pro 10.000 Geburten, wobei bei asiatischen, indianischen und hispanischen Kindern eine höhere Häufigkeit beobachtet wird [1-6].

Der Großteil der beobachteten Fälle von Schilddrüsendysgenesie wird als sporadisch angenommen, und die zugrunde liegenden Ursachen sind unklar. Bislang konnten nur für einen geringen Prozentsatz der PatientInnen mit familiärer Häufung pathogene Genvarianten identifiziert werden (vgl. - Tab. 2). Rezente Studien zeigen aber, dass, wenn nicht nur nach Kandidatengenen, sondern nach dem Phänotyp und auch unter Berücksichtigung ethnischer Gesichtspunkte genetische Analysen durchgeführt werden, ein stärkerer genetischer Hintergrund als bisher angenommen, vermutet werden kann [7].

TSH-Rezeptor und Transkriptionsfaktoren wie PAX8, NKX2-1 oder FOXE1 werden in der sich entwickelnden Schilddrüse exprimiert, und pathogene Varianten dieser Gene können nicht nur zur 
Tab. 1 Ursachen der kongenitalen Hypothyreose

Permanente primäre kongenitale Hypothyreose

\begin{tabular}{ll}
\hline Dysgenesie & Ektopie \\
& Athyreose \\
& $\begin{array}{l}\text { Hypoplasie/ } \\
\text { Hemiagenesie }\end{array}$ \\
Dyshormonogenese & $\begin{array}{l}\text { Schilddrüse normal } \\
\text { Struma }\end{array}$
\end{tabular}

Fehlentwicklung der Schilddrüse führen, sondern auch andere Gewebe bzw. Organe betreffen und so zur Symptomatik syndromaler Erkrankungen beitragen (Lungenerkrankungen, Chorea, Spaltbildungen). NKX2-1, zum Beispiel, ist wichtig für die Expression schilddrüsenspezifischer Gene (TG, TPO, TSHR oder SLC5a5 (Nis)) und reguliert auch die Expression von SP (Surfactantprotein) in der Lunge. Paired box gene 8 $(P A X 8)$ ist generell für die embryonale Entwicklung von Bedeutung und PAX8Mutationen wurden bereits in Familien mit kongenitaler Hypothyreose berichtet [8]. FOXE1 spielt nicht nur eine Rolle für die Entwicklung der Schilddrüse, sondern auch für Thymus und Pharynx. Eine FOXE1-Expression wird auch noch im Erwachsenenalter in der Schilddrüse, in der Epidermis und in Haarfollikeln beobachtet. Homozygote FOXE1-Mutationen wurden in Patienten mit dem Bamforth-Lazarus-Syndrom berichtet, das durch Dysgenesie der Schilddrüse, „spiky hair“ und eine Gaumenspalte gekennzeichnet ist. Ob FOXE1 tatsächlich kausal für die kongenitale Hypothyreose ist oder lediglich ein Suszeptibilitätsgen darstellt, kann kontrovers diskutiert werden [8-14].

Formen der Schilddrüsendyshormonogenese werden in den meisten Fällen autosomal rezessiv vererbt. Von Defekten betroffen sind Gene, die für die Schilddrüsenhormonsynthese essenziell sind, wobei je nach Gen die zusätzlich zur (immer vorhandenen) Hypothyreose auftretende Symptomatik durchaus unterschiedlich sein kann. Die kongenitale Hypothyreose wird entsprechend den Gendefekten auch im Rahmen von Syndromen (Pendred-Syndrom, etc.) beobachtet bzw. ist auch mit solchen (Down-

\begin{tabular}{|c|c|c|}
\hline Mögliche Gendefekte & OMIM & Assoziierte Syndrome \\
\hline NKX2-1/TFF1 NK2 homebox 1 & 600635 & Hirn-Lunge-Schilddrüse-Syndrom \\
\hline FOXE1/TTF2 Forkehead box 1 & 602617 & Bamforth-Syndrom \\
\hline PAX8 Paired box Gene 8 & 1674145 & Renale Abnormalitäten \\
\hline GLIS3 & 610192 & $\begin{array}{l}\text { Neonataler Diabetes, Glaukom, Polyzys- } \\
\text { tische Nieren }\end{array}$ \\
\hline JAG1 & 601920 & Alagille-Syndrom, Herzerkrankungen \\
\hline SLC26A4/PDS & 605646 & Sensorineurale Hörverluste \\
\hline TBX1 & 602054 & DiGeorge-Syndrom \\
\hline Dyshormonogenese & & OMIM \\
\hline Thyroperoxidase (TPO) & & 606765 \\
\hline Thyreoglobulin (TG) & & 188450 \\
\hline Dual Oxidase 2 (DUOX2) & & 606759 \\
\hline Natrium Jodid-Symporter Gen (SLC5A5, NIS) & & 601843 \\
\hline Deiodinase (IYD)/Dehalogenase (DEHAL) & & 612025 \\
\hline GNAS & & 139320 \\
\hline SLC26A4/PDS & & 605646 \\
\hline TSHR & & 603372 \\
\hline
\end{tabular}

Syndrom, Williams-Beuren-Syndrom) assoziiert (• Tab. 2).

\section{Indikationen für eine molekular- genetische Diagnostik}

Auch wenn die betroffenen PatientInnen mit einer entsprechenden Substitutionstherapie gut behandelt und betreut sind, ist eine genetische Diagnostik sinnvoll. Die Identifikation von pathogenen Genvarianten ist insbesondere bei familiärer Häufung, bei syndromalen Fällen oder PatientInnen mit unklaren neurologischen Defiziten von Relevanz, da nur bei Kenntnis des genetischen Hintergrunds der Erkrankung eine zuverlässige genetische Beratung (z.B. im Rahmen einer Familienplanung) betreffend Wiederholungsrisiko und Prognose angeboten werden kann.

Gerade die syndromalen Formen und die Tatsache, dass eine Reihe verschiedener Gene betroffen sein kann, erfordern, dass die genetische Diagnostik in auf diesem Gebiet erfahrenen Laboren bzw. Institutionen durchgeführt wird. Wie auch rezente Arbeiten zeigen, werden möglicherweise durch die rasant erfolgenden Weiterentwicklungen molekularbiologischer Methoden (NGS - next generation sequencing, whole exome oder clinical exome sequencing) weitere Genvarianten (bislang z.B. GLIS3, JAG1 oder
SLC26A4) identifiziert werden, die eine Rolle bei der Entstehung bzw. Ausprägung der primären kongenitalen Hypothyreose spielen [8-16].

Bevor eine humangenetische Analyse durch zuständige, einschlägige FachärztInnen veranlasst und im Labor durchgeführt werden kann, sind die PatientInnen entsprechend aufzuklären und zu beraten. Diese humangenetische Beratung muss dokumentiert werden, und die $\mathrm{Pa}$ tientInnen haben der Analyse schriftlich zuzustimmen. Das Ergebnis der genetischen Analyse muss in schriftlicher Form mitgeteilt und mit einer genetischen $\mathrm{Be}$ ratung abgeschlossen werden. Die PatientInnen können die Durchführung der humangenetischen Analyse bzw. die Mitteilung des Ergebnisses zu jedem Zeitpunkt und ohne Angabe von Gründen widerrufen.

Weitere Informationen über genetische Analysen in Österreich bekommen Sie unter anderem über folgenden Link: www.oeges.at (Molekulare Endokrinologie). 


\section{Korrespondenzadresse}

\section{Univ.-Prof. DI Dr. Sabina Baumgartner-}

\section{Parzer}

Universitätsklinik für Innere Medizin III, Medizinische Universität Wien

Währinger Gürtel 18-20, 1090 Wien, Österreich sabina.baumgartner-parzer@meduniwien.ac.at

Funding. Open access funding provided by Medical University of Vienna.

\section{Einhaltung ethischer Richtlinien}

Interessenkonflikt. S. Baumgartner-Parzer gibt an, dass kein Interessenkonflikt besteht.

Für diesen Beitrag wurden von den Autoren keine Studien an Menschen oder Tieren durchgeführt. Für die aufgeführten Studien gelten die jeweils dort angegebenen ethischen Richtlinien.

Open Access. Dieser Artikel wird unter der Creative Commons Namensnennung 4.0 International Lizenz (http://creativecommons.org/licenses/by/4.0/deed. de) veröffentlicht, welche die Nutzung, Vervielfältigung, Bearbeitung, Verbreitung und Wiedergabe in jeglichem Medium und Format erlaubt, sofern Sie den/die ursprünglichen Autor(en) und die Quelle ordnungsgemäßnennen, einen Linkzur Creative Commons Lizenz beifügen und angeben, ob Änderungen vorgenommen wurden.

\section{Literatur}

1. Persani L, Rurale G, deFilippis T, Galazzi E, Muzza M, Fugazzola L (2018) Genetics and management of congenital hypothyroidism. Best Pract Res Clin Endocrinol Metab 32:387-3396

2. Leger J, Olivieri A, Donaldson M et al (2014) European society for paediatric endocrinology consensus guidelines on screening diagnosis, and management of congenital hypthyroidism. J Clin Endocrinol Metab 99:363-384

3. Rastogi MV, LaFranchi SH (2010) Congenital hypothyroidism. Orphanet JRare Dis 5:17

4. American Academy of Pediatrics, Section on Endocrinology, Rose SR, Section on Endocrinology and Committee on Genetics, American Thyroid Association, Brown RS, Public Health Committee, Wilkins L et al (2006) Update of newborn screening and therapy for Congenital Hypothyroidism. Pediatr Electron Pages 117:2290-2303

5. Grüters A, Krude H (2011) Detection and treatment of congenital hypothyroidism. Nat Rev Endocrinol 18:104-113

6. Szinnai G (2012) Kongenitale Hypothyreose - ein Update über Diagnose, Therapie und Genetik. Paediatrica 23(5):12-15

7. Cherella CE, Wassner AJ (2017) Congenital hypothyroidism: Insights into pathogenesis and treatment.Int JPediatrEndocrinol.https://doi.org/ 10.1186/s13633-017-0051-0

8. Thorwarth A, Schnittert-Hübner S, Schrumpf P et al (2014) Comprehensive genotyping and clinical characterisation reveal 27 novel NKX2-1 mutations and expand the Phenotypic Spectrum. J Med Genet 51:375-387
9. Carvalho A, Hermanns $P$, Rodrigues $A L$ et al (2013) A new PAX8 mutation causing congenital hypothyroidism in three generations of a family is associated with abnormalities in the urogenital tract. Thyroid 23:1074-1078

10. Carre A, Szinnai G, Castanet M, Sura-Tureba S, Tron E, Broutin-L-Hermite I, Barat P, Goizet C, Lacombe D, Moutard ML, Raybaud C, Raynaud-Ravni C, Romana S, Ythier H, Leger J, Polak M (2009) Five new TTF1/NKX2.1 mutations in brain-lung-thyroid disease. Rescue by PAX8 synergism in one case. Hum Mol Genet 18:2266-2276

11. Trueba SS, Auge J, Mattei G et al (2005) PAX8, TITF1 and FOXE1 Gene Eexpression patterns during human development: new insights into human thyroid develompent and thyroid dysgenesisassociated malformations. J Clin Endocrinol Metab 90:455-456

12. Nicholas AK, Serra EG, Cangul $\mathrm{H}$ et al (2016) Comprehensive screening of eight known causative genes in congenital hypothyroidism with glandin-situ.J Clin Endocrinol Metab 101:4521-4531

13. DePhilippis T, Gelmini G, Parboschi E et al (2017) A frequent oligogenic involvement in congenital hypothyroidism. Hum Mol Genet 26:2507-2514

14. Park KJ, Park HK, Kim YJ et al (2016) DUOX2 mutations are frequently associated with congenital Hypothyroidism in the Korean population. Ann Lab Med 36:145-153

15. Fan X, Fu C, Shen Y, Li C, Luo S, Li Q et al (2017) Nextgeneration sequencing analysis of twelve known causative genes in congenital hypothyroidism. Clin Chim Acta 468:76-80

16. Carre A, Stoupa A, Kariyawasam D, Guerlouz M Ramond C, Monus T et al (2017) Mutations in BOREALIN cause Thyroid Dysgenesis. Hum Mol Genet 26:599-610

Hinweis des Verlags. Der Verlag bleibt in Hinblick auf geografische Zuordnungen und Gebietsbezeichnungen in veröffentlichten Karten und Institutsadressen neutral.

\section{Bluttest zur Früherkennung von Pankreaskrebs}

\section{Diabetespatienten für bundesweite} Studie gesucht

Mediziner des Greifswalder Pankreaszentrums an der Universitätsmedizin Greifswald haben gemeinsam mit Wissenschaftlern aus Kiel und Dresden und unter Beteiligung von 900 Patienten einen neuen Test entwickelt. Dieser ermöglicht es erstmals, anhand einer in Blutproben ermittelten sogenannten Metaboliten-Signatur, eines individuellen Profils von Stoffwechselprodukten, zwischen Gesunden, PankreatitisPatienten und Patienten mit Pankreaskarzinomen zu unterscheiden. Die Ergebnisse der Studie wurden 2017 in der renommierten internationalen Fachzeitschrift GUT veröffentlicht. Nun soll nach einer weiteren Verbesserung des Testsystems in einer bundesweiten Untersuchung an 20 Kliniken überprüft werden, ob sich eine Auswahl von zehn dieser Metaboliten in der klinischen Routine bewährt.

„Um festzustellen, ob sich der Test auch für die Erkennung von kleinen und frühen Tumoren eignet, werden 1500 Hochrisikopatienten untersucht", so der Direktor der Klinik für Innere Medizin A an der Unimedizin Greifswald, Prof. Dr. Markus M. Lerch. „Hierzu gehören nicht nur Patienten mit verdächtigen Röntgenbefunden der Bauchspeicheldrüse, sondern auch Frauen und Männer, die im Alter von über 50 Jahren erstmals an einem Diabetes mellitus erkranken. In den ersten 2 Jahren nach der Neuentdeckung eines Diabetes ist in dieser Altersgruppe das Risiko, ein Pankreaskarzinom zu entwickeln, 100 fach erhöht.

Für diese klinische Untersuchung zur Entdeckung von frühen Pankreaskarzinomen (META-PAC Studie) sucht das Studienzentrum der Klinik und Poliklinik für Innere Medizin A freiwillige Teilnehmer, bei denen im Alter von über 50 erstmals ein Diabetes diagnostiziert wurde. Interessenten sind herzlich eingeladen, sich unter der Telefonnummer 03834-86 7305 über die Teilnahmebedingungen zu informieren.

Quelle: Universität Greifswald, www.medizin.uni-greifswald.de 